\title{
Calculating propulsive and restoring electromagnetic forces between massive circular coils of rectangular cross section with inclined axes in air
}

\author{
Slobodan Babic ${ }^{1,}{ }^{*}$, Cevdet Akyel ${ }^{2}$ and Levent Erdogan 3 \\ ${ }^{1}$ Independent Researcher, 53 Berlioz 101, H3E 1N2, Montreal, Quebec, Canada. \\ ${ }^{2}$ École Polytechnique Montréal, C. P. 6079 Succ. Centre-Ville, QC H3C 3A7Quebec, Canada. \\ ${ }^{3}$ School of Sciences and Technology, University of the Azores, Ponta Delgada, Portugal.
}

World Journal of Advanced Engineering Technology and Sciences, 2021, 02(01), 110-121

Publication history: Received on 16 March 2021; revised on 17 April 2021; accepted on 19 April 2021

Article DOI: https://doi.org/10.30574/wjaets.2021.2.1.0032

\begin{abstract}
In this paper we give the new formulas for calculating the propulsive and the restoring electromagnetic forces between the loops in air whose centers are in the different axes. These new formulas are used to calculate the propulsive and the restoring forces between two inclined massive coils of rectangular cross section whose centers are in the different axes. The filament method is used. Results obtained by the presented approach are in remarkably good agreement with already published data. Electromagnetic forces can be used for various applications, it is very versatile; there are a plethora of ways to utilize electromagnetic forces and energy, from small scale uses e.g., microchips to larger scale and lifesaving uses e.g., radiotherapy.
\end{abstract}

Keywords: Mutual inductance; Propulsive and restoring electromagnetic forces; Inclined circular loops; Inclined massive coils of the rectangular cross section

\section{Introduction}

In electrical engineering the calculation of the magnetic forces acting on movable or immovable parts is of a great interest. The operation of some electromechanical and electromagnetic devices depends upon the forces that act either current-carrying conductors or magnetized parts. The calculation of the magnetic force and torque between two coils, carrying current, is a subject closely related to the calculation of their mutual inductance [1-3]. Today, it is possible to calculate the torque and magnetic force between inclined coils using the powerful numerical methods (FEM, BEM) which require a lot of computational time to achieve high accuracy (it is necessary to create a mesh in the model using enormous elements and nodes), [4-8]. Also, analytical, and semi-analytical methods are preferable, being more accurate and less time and memory consuming if they are applicable, [9-19]. In this paper we presented the simple approach to calculate the magnetic force between two inclined massive coils of the rectangular cross section where we use the filament method, [12-13]. Even though, in the literature there are not significant analytical and semi analytical methods regarding the subject of this paper we confirmed the results of this approach with those which are rare [14-20]. This approach could be especially useful in the design and optimization strategy for resonant electromagnetic vibration energy harvesting devices, torque sensors, transducers, actuators, and levitation systems.

\section{Basic expressions}

Let us take into consideration two inclined loops that carry currents of strength $I_{1}$ and $I_{2}$ (See Fig.1). The electromagnetic force $F$ between them [1-3] is,

\footnotetext{
* Corresponding author: Slobodan Babic

Independent Researcher, 53 Berlioz 101, H3E 1N2, Montreal, Quebec, Canada.
}

Copyright (C) 2021 Author(s) retain the copyright of this article. This article is published under the terms of the Creative Commons Attribution Liscense 4.0. 


$$
F=I_{1} I_{2} \frac{\partial M}{\partial g}
$$

where ' $g$ ' is the generalized coordinate, and the $M$ is the corresponding mutual inductance.

The mutual inductance $\boldsymbol{M}$ between two inclined filamentary circular loops one with radii $R_{P}$ and $R_{S}$ whose centers are not on the same axis (see Fig. 1), can be calculated as in [1],

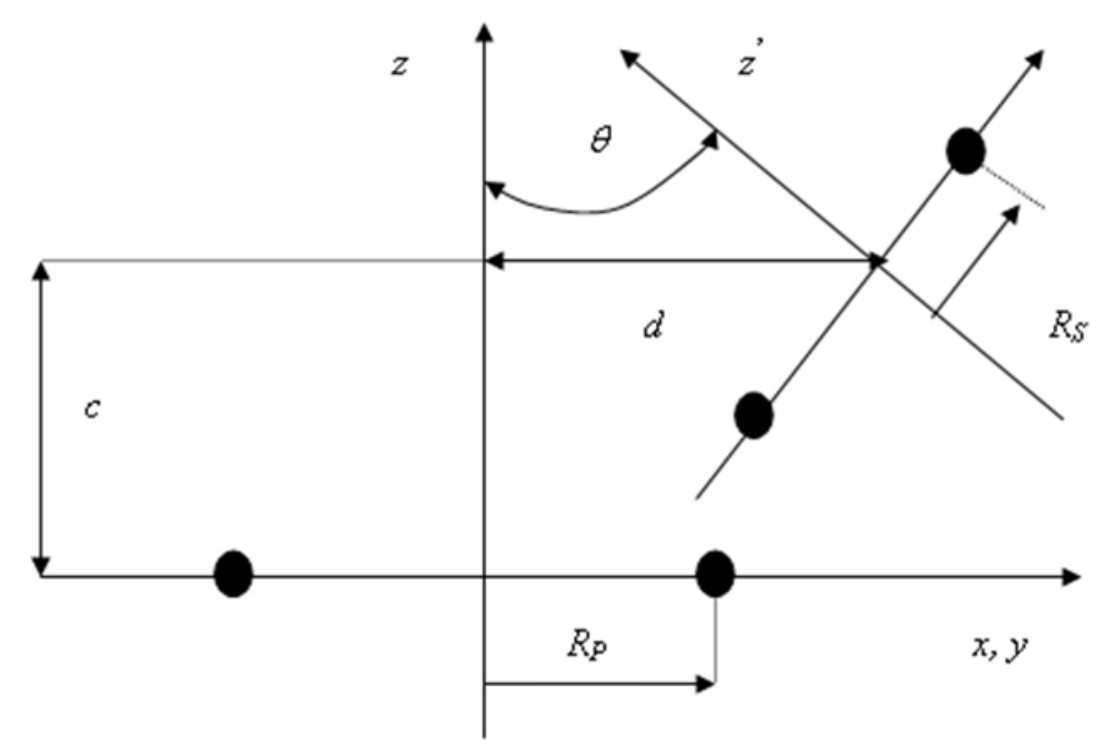

Figure 1 Filamentary circular loops with angular misalignment (axes intersect but not at the centre either of one).

$$
M=\frac{\mu_{0} \sqrt{R_{P} R_{S}}}{\pi} \int_{0}^{\pi} \frac{\left[\cos \theta-\frac{d}{R_{S}} \cos \phi\right] \Psi(k)}{\sqrt{V^{3}}} d \phi
$$

where

$$
\begin{gathered}
\alpha=\frac{R_{S}}{R_{P}}, \quad \beta=\frac{c}{R_{P}} \\
V=\sqrt{1-\cos ^{2} \phi \sin ^{2} \theta-2 \frac{d}{R_{S}} \cos \phi \cos \theta+\frac{d^{2}}{R_{S}^{2}}}, \quad k^{2}=\frac{4 \alpha V}{(1+\alpha V)^{2}+\xi^{2}} \\
\xi=\beta-\alpha \cos \phi \sin \theta, \quad \Psi(k)=\frac{1}{k}\left[\frac{2-k^{2}}{2} K(k)-E(k)\right]
\end{gathered}
$$

For the inclined filamentary circular loops (See Fig.1) the electromagnetic force F depends on the ' $c$ ' distance between loops' centres and ' $\boldsymbol{d}$ ' distance between axes because the axes of inclined loops axes intersect but not at the centre of either one so that the electromagnetic force $\mathrm{F}$ is given by the axial (propulsive) force and the radial (restoring) force which are calculated by,

$$
\begin{aligned}
& F_{\text {propulsive }}=I_{1} I_{2} \frac{\partial M}{\partial c}=F_{\text {axial }}=F_{c} \\
& F_{\text {restoring }}=I_{1} I_{2} \frac{\partial M}{\partial d}=F_{\text {radial }}=F_{d}
\end{aligned}
$$


Finding the first derivatives of $M$ given from (1) by using (2), (3) and (4) the axial (propulsive) and the radial (restoring) electromagnetic forces are respectively,

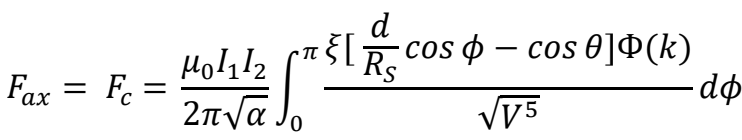

$F_{r a}=F_{d}=\frac{\mu_{0} I_{1} I_{2}}{\pi \sqrt{\alpha}} \int_{0}^{\pi}\left[\frac{d^{2}}{R_{S}^{2}} \cos \phi+\frac{d}{R_{S}} \cos \theta\left(\cos ^{2} \phi-3\right)+\cos \phi\left(1+2 \cos ^{2} \phi \sin ^{2} \theta-\right.\right.$

$\left.\left.-3 \sin ^{2} \theta\right)\right] \frac{\Phi(k)}{\sqrt{V^{7}}} d \phi-\frac{\mu_{0} I_{1} I_{2}}{4 \pi \alpha \sqrt{\alpha}} \int_{0}^{\pi}\left(\frac{d}{R_{S}} \cos \phi-\cos \theta\right)\left(\frac{d}{R_{S}}-\cos \theta \cos \phi\right)(1+$

$\left.+\xi^{2}-\alpha^{2} V^{2}\right) \frac{\Psi(k)}{\sqrt{V^{9}}} d \phi$

where $\alpha, \beta, \mathrm{V}, \mathrm{k} 2, \xi, \Psi(\mathrm{k})$ are previously given and

$$
\Phi(k)=k\left[\frac{2-k^{2}}{2\left(1-k^{2}\right)} E(k)-K(k)\right]
$$

$R_{P}$ - radius of the primary loop (larger loop).

$R_{S}$ - radius of the secondary loop (smaller loop).

$c$ - distance between coils' centers.

$d$ - distance between axes.

$\theta$ - angle between coil planes.

$I_{1}$ - current in the primary loop.

$I_{2}$ - current in the secondary loop.

$K(k)$ - complete elliptic integral of the first kind [21-22].

$E(k)$ - complete elliptic integral of the second kind [21-22].

$\mu_{0}=4 \pi \cdot 10-7 \mathrm{H} / \mathrm{m}$ - magnetic permeability of vacuum.

The formulas (5) and (6) appear for the first time in the literature. It is not difficult to show that in the limit case where circular loops are in the different parallel planes, expressions (5) and (6) become the same as those in [18].

\section{Propulsive and restoring electromagnetic forces between two thick inclined circular coils of rectangular cross section}

In [14], we calculated the mutual inductance between the thick circular coils of rectangular cross section with inclined axes (See Fig.2) using the filament method.

Let us consider the system comprising two circular coils of rectangular cross section with $\mathrm{N}_{1}, N_{2}$ turns, respectively. The centers of these coils are on the different axes (Fig.2). The centers of the inclined filamentary coils that replace the secondary coil do not have the same distances.

from the axis of the primary coil either for the inclined coil with centers on the primary axis or for the inclined coils with centers on the secondary axis. Using the same logic as in [12-13] the electromagnetic forces between two inclined circular coils of rectangular cross section (centers are in the different axes, Fig.1) can be obtained in the following form,

$$
F_{\text {propulsive }}=\frac{N_{1} N_{2} \sum_{g=-K}^{g=K} \sum_{h=-N}^{h=N} \sum_{l=-n}^{l=n} \sum_{p=-m}^{p=m} F_{c}(g, h, l, p)}{(2 K+1)(2 N+1)(2 n+1)(2 m+1)}
$$




$$
F_{\text {restoring }}=\frac{N_{1} N_{2} \sum_{g=-K}^{g=K} \sum_{h=-N}^{h=N} \sum_{l=-n}^{l=n} \sum_{p=-m}^{p=m} F_{d}(g, h, l, p)}{(2 K+1)(2 N+1)(2 n+1)(2 m+1)}
$$

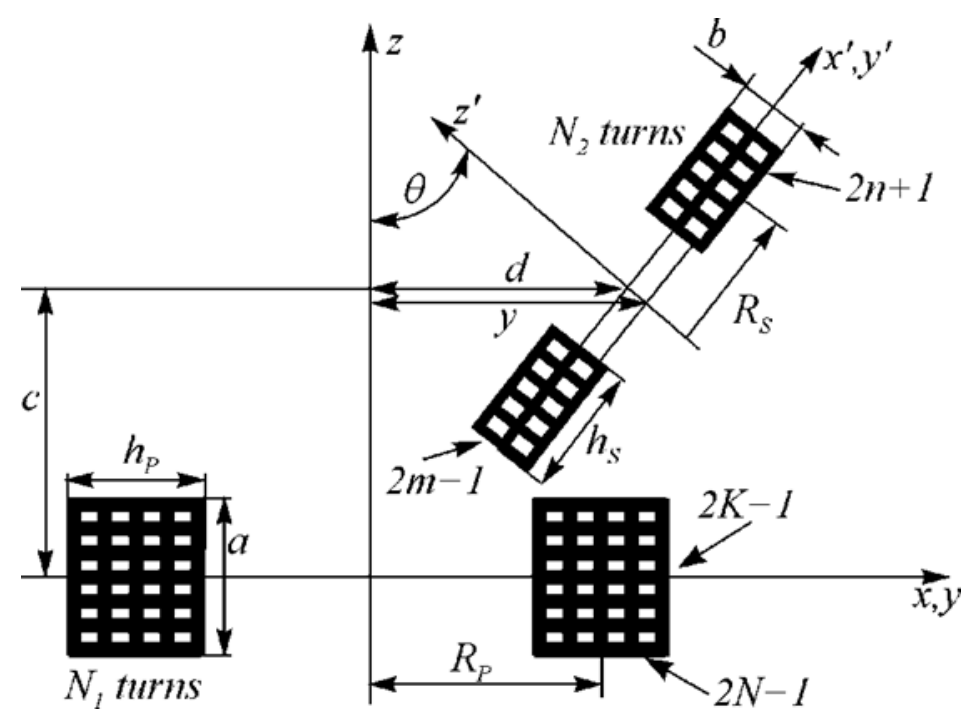

Figure 2 Configuration of mesh matrix: Two circular coils of rectangular cross section (axes intersect but not at the centre of either one).

Where

$F_{a x}(g, \boldsymbol{h}, l, p)=\frac{\mu_{0} I_{1} I_{2}}{2 \pi \sqrt{\alpha}} \int_{0}^{\pi} \frac{\xi\left[\frac{y(p)}{R_{S}(l)} \cos \phi-\cos \theta\right] \Phi(k)}{\sqrt{V^{5}}} d \phi$
$F_{r a}(g, \boldsymbol{h}, l, p)=\frac{\mu_{0} I_{1} I_{2}}{\pi \sqrt{\alpha}} \int_{0}^{\pi}\left[\frac{y^{2}(p)}{R_{S}^{2}(l)} \cos \phi+\frac{y(p)}{R_{S}(l)} \cos \theta\left(\cos ^{2} \phi-3\right)+\cos \phi(1+\right.$

$\left.\left.+2 \cos ^{2} \phi \sin ^{2} \theta-3 \sin ^{2} \theta\right)\right] \frac{\Phi(k)}{\sqrt{V^{7}}} d \phi-\frac{\mu_{0} I_{1} I_{2}}{4 \pi \alpha \sqrt{\alpha}} \int_{0}^{\pi}\left(\frac{y(p)}{R_{S}(l)} \cos \phi-\cos \theta\right)\left(\frac{y(p)}{R_{S}(l)}\right.$

$$
\begin{gathered}
-\cos \theta \cos \phi)\left(1+\xi^{2}-\alpha^{2} V^{2}\right) \frac{\Psi(k)}{\sqrt{V^{9}}} d \phi \\
\alpha(\square, l)=\frac{R_{S}(h)}{R_{P}(l)}, \quad \beta(g, p)=\frac{z(g, p)}{R_{P}} \\
V(l, p)=\sqrt{1-\cos ^{2} \phi \sin ^{2} \theta-2 \frac{y(p)}{R_{S}(l)} \cos \phi \cos \theta+\frac{y^{2}(p)}{R_{S}^{2}(l)}} \\
\xi(g, \boldsymbol{h}, l, p)=\beta-\alpha \cos \phi \sin \theta
\end{gathered}
$$




$$
\begin{gathered}
k^{2}(g, \square, l, p)=\frac{4 \alpha V}{(1+\alpha V)^{2}+\xi^{2}} \\
y_{0}(p)=\frac{b}{(2 m+1)} p ; p=-m, \ldots, 0, \ldots, m \\
y(p)=d+y_{0}(p) \sin \theta \\
z(g, p)=c+\frac{a}{(2 K+1)} g-y_{0}(p) \cos \theta \\
g=-K, \ldots, 0, \ldots, K ; p=-m, \ldots, 0, \ldots, m \\
R_{P}(h)=R_{P}+\frac{h_{P}}{(2 N+1)} h ; h=-N, \ldots, 0, \ldots, N ; \quad R_{P}=\frac{R_{1}+R_{2}}{2}, h_{P}=R_{2}-R_{1} \\
R_{S}(l)=R_{S}+\frac{h_{S}}{(2 n+1)} l ; l=-n, \ldots, 0, \ldots, n ; \quad R_{S}=\frac{R_{3}+R_{4}}{2}, h_{S}=R_{4}-R_{3}
\end{gathered}
$$

$\Psi(k)$ and $\Phi(k)$ are previously given.

$R_{1}$ - inner radius of the primary coil of rectangular cross section.

$R_{2}$ - outer radius of the primary coil of rectangular cross section.

$R_{3}$ - inner radius of the secondary coil of rectangular cross section.

$R_{4}$ - outer radius of the secondary coil of rectangular cross section.

$a$ and $b$ - heights of the primary and the secondary coil, respectively.

$I_{1}$ - current in the primary coil.

$I_{2}$ - current in the secondary coil.

$N_{1}$ - number of turns in the primary coil.

$N_{2}$ - number of turns in the secondary coil.

$R P(h)$ - average radius of the primary coil (larger coil) positioned in the plane $(x, y)$ whose axis is ' $z$ ' axis.

$R_{S}(l)$ - inclined average radius of the secondary coil (smaller coil)

$K, N, n$ and $m$ are the number of the subdivisions of thick coils.

Obviously from the Figure 2 that distance $z(g, p)$ between the distance $y(p)$ between axes is variable.

From this general case it is possible to calculate the electromagnetic force between any two possible inclined coils for which equations (5) and (6) must be obligatorily applied, for example, between two inclined thin wall solenoids, between two inclined pancakes and between all combinations of the massive coils, thin wall solenoids, pancakes and the circular coils.

In all calculation either for two thick inclined circular coils of rectangular cross section with equations (7) and (8) we treat only non-overlapping cases. In the case of overlapping coils there are the logarithmic singularities, and the convergence is limited in this domain. It is linked to physics so that these cases are not interested in this approach. Thus, in this paper we treat only regular and practical cases where coils do not overlap.

\section{Numerical validation}

Even though there is not a lot of papers in the literature concerning the previously mentioned forces we use some published results as the special cases which can validate our approach. We compared the results obtained by different methods.

\subsection{Example 1}

Calculate the propulsive and the restoring forces for two circular loops of radii $\boldsymbol{R}_{\mathbf{1}}=1(\mathrm{~m})$ and $\boldsymbol{R}_{\mathbf{2}}=0.5(\mathrm{~m})$. Loops lie in parallel planes $z_{1}(\mathrm{~m})$ and $z_{2}(\mathrm{~m})$, respectively $\left(\mathbf{z}_{1}-\mathbf{z}_{\mathbf{2}}=\mathrm{c}\right)$ and their axes are separated by the distance $\boldsymbol{d}(\mathrm{m})$. Both loops carry a current of $1(\mathrm{~A}),[15]$. 
In this example we compare the results obtained by this work (Formulas (5) and (6)) with those obtained in [15]. The noncoplanar loops are with the parallel axes. There is not the analytical solution for the restoring and propulsive electromagnetic forces given by (5) and (6) as well as given by [15]. In this work, the forces are obtained by the simple integration of the elementary analytical function and in [15] by the Bessel integrals.

Table 1 Restoring and propulsive magnetic forces. Case $\cos (\boldsymbol{\theta})=1$.

\begin{tabular}{|c|c|c|c|c|c|}
\hline $\boldsymbol{d}(\mathbf{m})$ & $\boldsymbol{c}(\mathbf{m})$ & $F_{r a}(\boldsymbol{\mu H}),[\mathbf{1 5}]$ & $F_{a x}(\boldsymbol{\mu H}),[\mathbf{1 5}]$ & $F_{\boldsymbol{r a}}(\boldsymbol{\mu H}),(\mathbf{5})$ & $\boldsymbol{F}_{\boldsymbol{a x}}(\boldsymbol{\mu H}),(\mathbf{6})$ \\
\hline 0.25 & 0.01 & 0.41346695105714 & -0.4298995175276 & 0.41346695105714 & -0.4298995175276 \\
\hline 0.25 & 0.10 & 0.32584843375637 & -0.3854548445797 & 0.32584843375637 & -0.3854548445797 \\
\hline 0.25 & 1.00 & -0.052206562874 & -0.2373062370517 & -0.052206562874 & -0.2373062370517 \\
\hline 0.5 & 0.01 & 4.18902497773124 & -6.2588817210507 & 4.18902497773124 & -6.2588817210507 \\
\hline 0.5 & 0.10 & 0.21943937237365 & -1.8641812311391 & 0.21943937237365 & -1.8641812311391 \\
\hline 0.5 & 1.00 & -0.099830101523 & -0.203312433039 & -0.099830101523 & -0.203312433039 \\
\hline 1.0 & 0.01 & -1.357468443781 & -0.318603560562 & -1.357468443781 & -0.318603560562 \\
\hline 1.0 & 0.10 & -1.115107228517 & -0.270826141873 & -1.115107228517 & -0.270826141873 \\
\hline 1.0 & 1.00 & -0.135254628361 & -0.076941561504 & -0.135254628361 & -0.076941561504 \\
\hline 1.5 & 0.01 & 2.5588394720979 & 3.5443981627819 & 2.5588394720979 & 3.5443981627819 \\
\hline 1.5 & 0.10 & 0.2235498200423 & 0.9168227297951 & 0.2235498200423 & 0.9168227297951 \\
\hline 1.5 & 1.00 & -0.074721057743 & 0.0190514847824 & -0.074721057743 & 0.0190514847824 \\
\hline 2.0 & 0.01 & 0.1035570996265 & 0.0027693210081 & 0.1035570996265 & 0.0027693210081 \\
\hline 2.0 & 0.10 & 0.0974888286519 & 0.0265606886035 & 0.0974888286519 & 0.0265606886035 \\
\hline 2.0 & 1.00 & -0.017526251790 & 0.0288410475404 & -0.017526251790 & 0.0288410475404 \\
\hline
\end{tabular}

Table 1 shows the identical results obtained by two different methods. All treated cases are the regular cases (loops do not overlap or touch).

\subsection{Example 2}

Let us calculate the restoring and the propulsive electromagnetic forces for two inclined loops for which is $\cos (\theta)=$ $0.5 \sqrt{3} ; 0.5 \sqrt{2} ; 0.5$ and 0.0 , successively. The loops are with radii $R_{P}=2 \mathrm{~m}$ and $R s=1 \mathrm{~m}$ respectively and $c=2(\mathrm{~m}), 0<d$ $<5 \mathrm{~m}$.

a) $\cos (\theta)=0.5 \sqrt{3}$ or $\theta=\pi / 6$. The loops are inclined. From [19] we use two loops with inclined axes where the primary loop is in the plane $z=0$ and the secondary loop in the plane $-y+\sqrt{3} z=0, \quad x_{c}=0,0 \leq y_{c} \leq$ $6(\mathrm{~m}), z_{c}=c=2(\mathrm{~m})$. The unit vector on the secondary loop is $\vec{N}=\{a, b, c\}=\{0,-0.5,0.5 \sqrt{3}\}$. For this case, the comparative results obtained by this work and [20]. Results are identical, (Table 2). 
Table 2 Restoring and propulsive magnetic forces. Case $\cos (\theta)=0.5 \sqrt{3}$.

\begin{tabular}{|c|c|c|c|c|c|}
\hline $\boldsymbol{d}(\mathbf{m})$ & $\boldsymbol{c}(\mathbf{m})$ & $F_{r a}(\boldsymbol{\mu H}),[\mathbf{1 9 ]}$ & $F_{a x}(\boldsymbol{\mu H}),[\mathbf{1 9 ]}$ & $F_{r a}(\boldsymbol{\mu H}),(\mathbf{5})$ & $F_{a x}(\boldsymbol{\mu H}),(\mathbf{6})$ \\
\hline 0.0 & 2.0 & -0.058717526303 & -0.224114257915 & -0.058717526303 & -0.224114257915 \\
\hline 0.5 & 2.0 & -0.098234539667 & -0.189792506531 & -0.098234539667 & -0.189792506531 \\
\hline 1.0 & 2.0 & -0.128626521630 & -0.143313137001 & -0.128626521630 & -0.143313137001 \\
\hline 1.5 & 2.0 & -0.144598582384 & -0.082024100496 & -0.144598582384 & -0.082024100496 \\
\hline 2.0 & 2.0 & -0.134771421954 & -0.012306272231 & -0.1347714421954 & -0.012306272231 \\
\hline 2.5 & 2.0 & -0.094525885335 & 0.044583441668 & -0.094525885335 & 0.044583441668 \\
\hline 3.0 & 2.0 & -0.041491415171 & 0.065994683081 & -0.041491415171 & 0.065994683081 \\
\hline 3.5 & 2.0 & -0.003817386333 & 0.055994117083 & -0.003817386333 & 0.055994117083 \\
\hline 4.0 & 2.0 & 0.011666608017 & 0.037344022297 & 0.011666608017 & 0.037344022297 \\
\hline 4.5 & 2.0 & 0.014555255204 & 0.022694295428 & 0.014555255204 & 0.022694295428 \\
\hline 5.0 & 2.0 & 0.012972391481 & 0.013483668023 & 0.012972391481 & 0.013483668023 \\
\hline 5.5 & 2.0 & 0.010456213799 & 0.008059497539 & 0.010456213799 & 0.008059497539 \\
\hline 6.0 & 2.0 & 0.008144754459 & 0.004892823977 & 0.008144754459 & 0.004892823977 \\
\hline
\end{tabular}

b) $\cos (\theta)=0.5 \sqrt{2}$ or $\theta=\pi / 4$. The loops are inclined. From [19] we use two loops with inclined axes where the primary loop is in the plane $z=0$ and the secondary loop in the plane $-y+z=0, x_{c}=0,0 \leq y_{c} \leq 6(\mathrm{~m})$, $z_{c}=c=2(\mathrm{~m})$. The unit vector on the secondary loop is $\vec{N}=\{a, b, c\}=\{0,-1,1\}$. For this case, the comparative results obtained by this work and [20]. Results are identical. (Table 3 ).

Table 3 Restoring and propulsive magnetic forces. Case $\cos (\theta)=\mathbf{0 . 5} \sqrt{\mathbf{2}}$.

\begin{tabular}{|c|c|c|c|c|c|}
\hline $\boldsymbol{d}(\mathbf{m})$ & $\boldsymbol{c}(\mathbf{m})$ & $F_{r a}(\boldsymbol{\mu H}),[\mathbf{1 9 ]}$ & $F_{a x}(\boldsymbol{\mu H}),[\mathbf{1 9 ]}$ & $\boldsymbol{F}_{r a}(\boldsymbol{\mu H}),(\mathbf{5})$ & $\boldsymbol{F}_{\boldsymbol{a x}}(\boldsymbol{\mu H}),(\mathbf{6})$ \\
\hline 0.0 & 2.0 & -0.08906461894 & -0.188372647312 & -0.08906461894 & -0.188372647312 \\
\hline 0.5 & 2.0 & -0.117662588147 & -0.146589320523 & -0.117662588147 & -0.146589320523 \\
\hline 1.0 & 2.0 & -0.138868519142 & -0.097006617699 & -0.138868519142 & -0.097006617699 \\
\hline 1.5 & 2.0 & -0.146421506732 & -0.033540198539 & -0.146421506732 & -0.033540198539 \\
\hline 2.0 & 2.0 & -0.125005785102 & 0.037703090907 & -0.125005785102 & 0.037703090907 \\
\hline 2.5 & 2.0 & -0.06846447843 & 0.087566122674 & -0.06846447843 & 0.087566122674 \\
\hline 3.0 & 2.0 & -0.000740119765 & 0.08822211306 & -0.000740119765 & 0.08822211306 \\
\hline 3.5 & 2.0 & 0.022728697047 & 0.05976143639 & 0.022728697047 & 0.05976143639 \\
\hline 4.0 & 2.0 & 0.027395133109 & 0.03391261801 & 0.027395133109 & 0.03391261801 \\
\hline 4.5 & 2.0 & 0.023109885214 & 0.01818579088 & 0.023109885214 & 0.01818579088 \\
\hline 5.0 & 2.0 & 0.017624821231 & 0.00964636754 & 0.017624821231 & 0.00964636754 \\
\hline 5.5 & 2.0 & 0.013056738094 & 0.00510944579 & 0.013056738094 & 0.00510944579 \\
\hline 6.0 & 2.0 & 0.009645009493 & 0.00268103227 & 0.009645009493 & 0.00268103227 \\
\hline
\end{tabular}

c) $\cos (\theta)=0.5$ or $\theta=\pi / 3$. The loops are inclined. From [19] we use two loops with inclined axes where the primary loop is in the plane $z=0$ and the secondary loop in the plane $-\sqrt{3} y+z=0, \quad x_{c}=0,0 \leq y_{c} \leq$ $6(\mathrm{~m}), z_{c}=c=2(\mathrm{~m})$. The unit vector on the secondary loop is $\vec{N}=\{a, b, c\}=\{0,-\sqrt{3}, 1\}$. For this case, the comparative results obtained by this work and [20]. Results are identical, (Table 4). 
Table 4 Restoring and propulsive magnetic forces. Case $\cos (\theta)=\mathbf{0 . 5}$.

\begin{tabular}{|c|c|c|c|c|c|}
\hline $\boldsymbol{d}(\mathbf{m})$ & $\boldsymbol{c}(\mathbf{m})$ & $\boldsymbol{F}_{\boldsymbol{r a}}(\boldsymbol{\mu H}),[\mathbf{1 9 ]}$ & $\boldsymbol{F}_{\boldsymbol{a x}}(\boldsymbol{\mu H}),[\mathbf{1 9 ]}$ & $\boldsymbol{F}_{\boldsymbol{r a}}(\boldsymbol{\mu H}),(\mathbf{5})$ & $\boldsymbol{F}_{\boldsymbol{a x}}(\boldsymbol{\mu H}),(\mathbf{6})$ \\
\hline 0.0 & 2.0 & -0.1137924691470 & -0.1349975514965 & -0.1137924691470 & -0.1349975514965 \\
\hline 0.5 & 2.0 & -0.1310477690771 & -0.0905018241289 & -0.1310477690771 & -0.0905018241289 \\
\hline 1.0 & 2.0 & -0.1434308001709 & -0.0388937270022 & -0.1434308001709 & -0.0388937270022 \\
\hline 1.5 & 2.0 & -0.1403249232461 & 0.0279696206824 & -0.1403249232461 & 0.0279696206824 \\
\hline 2.0 & 2.0 & -0.1000071650655 & 0.0988741066612 & -0.1000071650655 & 0.0988741066612 \\
\hline 2.5 & 2.0 & -0.0227155175608 & 0.1279529527325 & -0.0227155175608 & 0.1279529527325 \\
\hline 3.0 & 2.0 & 0.0344759827275 & 0.0958379634733 & 0.0344759827275 & 0.0958379634733 \\
\hline 3.5 & 2.0 & 0.0462744888982 & 0.0526151474770 & 0.0462744888982 & 0.0526151474770 \\
\hline 4.0 & 2.0 & 0.0384565415946 & 0.0255697243523 & 0.0384565415946 & 0.0255697243523 \\
\hline 4.5 & 2.0 & 0.0282062890787 & 0.0117981616862 & 0.0282062890787 & 0.0117981616862 \\
\hline 5.0 & 2.0 & 0.0200269531157 & 0.0051334161394 & 0.0200269531157 & 0.0051334161394 \\
\hline 5.5 & 2.0 & 0.0142064646411 & 0.0019394489162 & 0.0142064646411 & 0.0019394489162 \\
\hline 6.0 & 2.0 & 0.0101868302564 & 0.0004216609096 & 0.0101868302564 & 0.0004216609096 \\
\hline
\end{tabular}

d) $\cos (\theta)=0.0$ or $\theta=\pi / 2$. The loops are orthogonal mutually. From [19] we use two loops with inclined axes where the primary loop is in the plane $z=0$ and the secondary loop in the plane $-y=0, x_{c}=0,0 \leq y_{c} \leq 6(\mathrm{~m}), z_{c}=c=$ 2 (m). The unit vector on the secondary loop is $\vec{N}=\{a, b, c\}=\{0,-1,0\}$. For this case, the comparative results obtained by this work and [20]. Results are identical, (Table5).

Table 5 Restoring and propulsive magnetic forces. Case $\cos (\theta)=0.0$.

\begin{tabular}{|c|c|c|c|c|c|}
\hline $\boldsymbol{d}(\mathbf{m})$ & $\boldsymbol{c}(\mathbf{m})$ & $F_{r a}(\boldsymbol{\mu H}),[19]$ & $F_{a x}(\boldsymbol{\mu H}),[\mathbf{1 9 ]}$ & $F_{r a}(\boldsymbol{\mu H}),(5)$ & $F_{a x}(\boldsymbol{\mu H}),(\mathbf{6})$ \\
\hline 0.0 & 2.0 & 0.1334313173181 & 0.0 & -0.1334313173181 & 0.0 \\
\hline 0.5 & 2.0 & 0.1327811215389 & -0.0460078388114 & -0.1327811215389 & 0.0460078388114 \\
\hline 1.0 & 2.0 & 0.1225753397431 & -0.1050825924157 & -0.1225753397431 & 0.1050825924157 \\
\hline 1.5 & 2.0 & 0.0761025506837 & -0.1733955108217 & -0.0761025506837 & 0.1733955108217 \\
\hline 2.0 & 2.0 & -0.019349551084 & -0.1947429489301 & 0.019349551084 & 0.1947429489301 \\
\hline 2.5 & 2.0 & -0.0859302495866 & -0.1323768968367 & 0.0859302495866 & 0.1323768968367 \\
\hline 3.0 & 2.0 & -0.0864305037745 & -0.0634927535303 & 0.0864305037745 & 0.0634927535303 \\
\hline 3.5 & 2.0 & -0.0644722369249 & -0.0249227437307 & 0.0644722369249 & 0.0249227437307 \\
\hline 4.0 & 2.0 & -0.0439958709655 & -0.0073755395766 & 0.0439958709655 & 0.0073755395766 \\
\hline 4.5 & 2.0 & -0.0293694111013 & -0.0001097549547 & 0.0293694111013 & 0.0001097549547 \\
\hline 5.0 & 2.0 & -0.0196674446246 & 0.000257758497 & 0.0196674446246 & -0.000257758497 \\
\hline 5.5 & 2.0 & -0.0133475906431 & 0.000331382874 & 0.0133475906431 & -0.000331382874 \\
\hline 6.0 & 2.0 & -0.0009217011890 & 0.000326186324 & 0.0009217011890 & -0.000326186324 \\
\hline
\end{tabular}

From Tables 2-5 we show the validity of the new formulas (5) and (6). Results obtained by [19] and this work are identical that is expected. Also, the same results can be obtained by [20]. 


\subsection{Example 3}

In [18], we calculate the magnetic force between two misalignment superconducting coils of rectangular cross section with the following parameters: $R_{1}=0.07124(\mathrm{~m}), R_{2}=0.0969645(\mathrm{~m}), R_{3}=0.085217(\mathrm{~m}), R_{4}=0.1384935(\mathrm{~m}), a=$ $0.142748(\mathrm{~m}), \mathrm{b}=0.02413(\mathrm{~m}), N_{1}=1142, N_{2}=516$. In each coil the operating current is 1 (A), [13]. The axial displacement is $c=0$. The coils are with parallel axes. Let us present graphically the propulsive and the restoring forces for $\cos (\theta)=0.0 ; 0.1 ; 0.2 ; 0.3 ; 0.4 ; 0.5 ; 0.6 ; 0.7 ; 0.8 ; 0.9$ and 1.0 , where $c=4(\mathrm{~m})$ and $d=0$ to $8(\mathrm{~m})$.

The propulsive magnetic force is equal zero because the axial displacement is $c=0$. Thus, we calculate the transverse magnetic force due to the asymmetry. This force is also called the radial or restoring force because of the misalignment between coil axes. The formula (8) and the filament method are used to calculate this transverse force. The coils of the rectangular cross sections are with the parallel axes so that $\cos (\theta)=1.0$.

Table 6 Radial force as a function of the perpendicular displacement $\mathrm{d}$ of two coil axes for the plans displacement $\mathrm{c}=0$. $(\theta=0.0)$.

\begin{tabular}{|c|c|c|c|}
\hline $\boldsymbol{d}(\mathbf{m})$ & $\begin{array}{c}\text { Radial force [13] } \\
\boldsymbol{F}_{\boldsymbol{r}} \mathbf{( m \mathbf { ) } )}\end{array}$ & $\begin{array}{c}\text { Radial force (7), [18] } \\
\boldsymbol{F}_{\boldsymbol{r}} \mathbf{( m N )}\end{array}$ & $\begin{array}{c}\text { Radial force (This work } \mathbf{( 8 )}) \\
\boldsymbol{F}_{\boldsymbol{R}}(\mathbf{m N} \mathbf{)}\end{array}$ \\
\hline 0.00 & 0.00 & 0.00 & 0.00 \\
\hline 0.001 & 3.40505 & 3.40458990 & 3.40458990 \\
\hline 0.002 & 6.80992 & 6.80877851 & 6.80877851 \\
\hline 0.003 & 10.2145 & 10.21216381 & 10.21216381 \\
\hline 0.004 & 13.6185 & 13.61433904 & 13.61433904 \\
\hline 0.005 & 17.0217 & 17.01490137 & 17.01490137 \\
\hline 0.006 & 20.4239 & 20.41342339 & 20.41342339 \\
\hline 0.007 & 23.8246 & 23.80949684 & 23.80949684 \\
\hline 0.008 & 27.2312 & 27.20265102 & 27.20265102 \\
\hline 0.009 & 30.6202 & 30.59236145 & 30.59236145 \\
\hline 0.010 & 34.0141 & 33.97781178 & 33.97781178 \\
\hline 0.011 & 37.4045 & 37.35721526 & 37.35721526 \\
\hline
\end{tabular}

Equations (7) in [18] and (8) from this work give identical results and they are in particularly good agreement with those obtain by the approach in [13]. In [13], the radial magnetic force has been obtained by the double numerical integration and in this work by the single numerical integration.

Applying (7) and (8) for $\cos (\theta)=0.0 ; 0.1 ; 0.2 ; 0.3 ; 0.4 ; 0.5 ; 0.6 ; 0.7 ; 0.8 ; 0.9$ and 1.0 , where $\boldsymbol{c}=4(\mathrm{~m})$ and $\boldsymbol{d}=0$ to $8(\mathrm{~m})$ we give Figures 3 and 4, which show graphically the propulsive and the restoring electromagnetic forces for different angles of the inclination of the missive coils of rectangular cross section. 


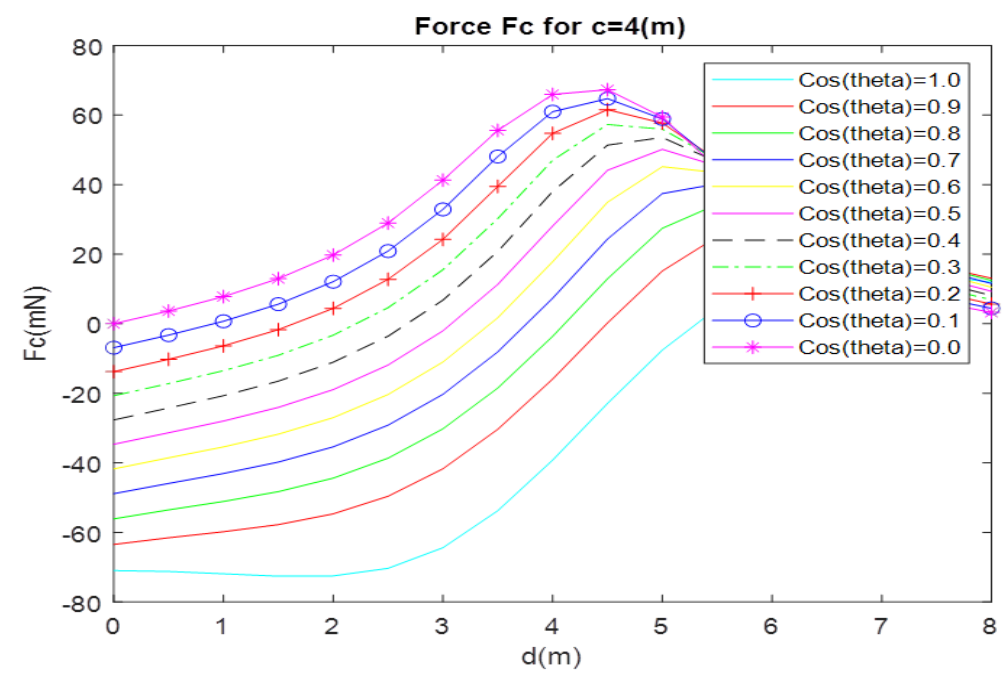

Figure 3 The axial (propulsive) electromagnetic force $\mathrm{Fc}$ for the different angle $\theta$ and the different distance d, (Inclined massive coils of the rectangular cross section).

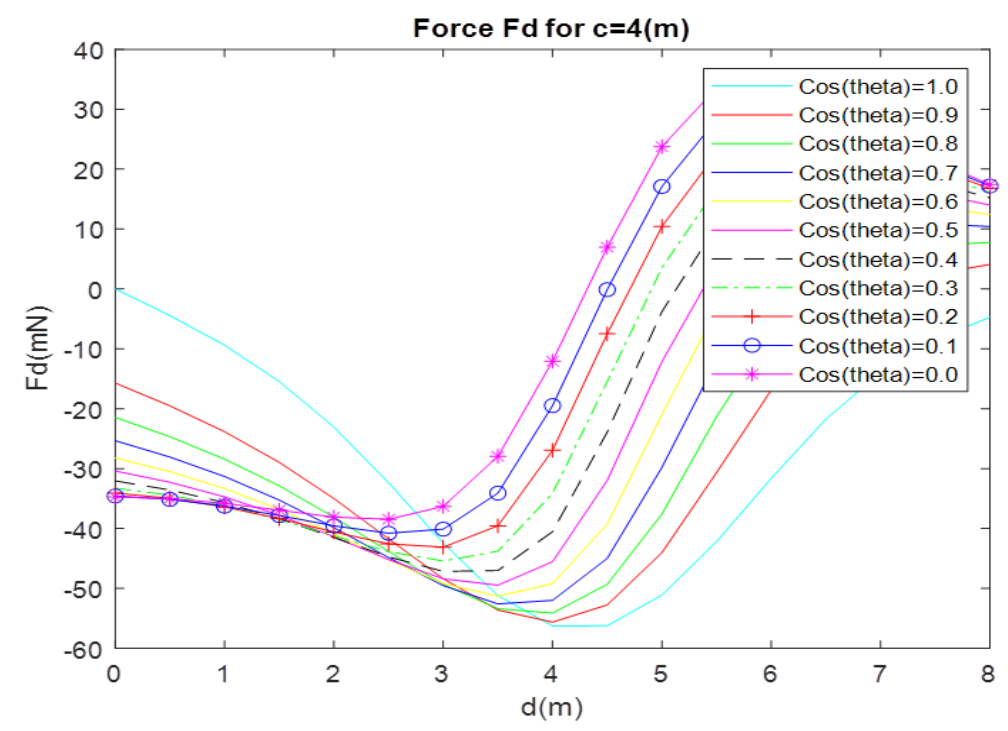

Figure 4 The radial (restoring) electromagnetic force $\mathrm{Fd}$ for the different angle $\theta$ and the different distance $\mathrm{d}$, (Inclined massive coils of the rectangular cross section).

\section{Conclusion}

In this paper, we propose two new formulas for calculation the propulsive and the restoring electromagnetic forces between circular loops with misaligned axes regarding the axial and radial displacement. Using the filament method and these two new formulas we give expressions of electromagnetic forces (axial and radial) between two inclined massive circular coils of the rectangular cross section. From these expressions it is possible to calculate the electromagnetic forces between all possible inclined circular configurations (massive coils, thin wall solenoids, thin pancakes and the filamentary coils). The presented method can be used in the large scale of practical applications either for micro coils or for large coils. Presented examples show that all results obtained by the presented approach are in an excellent agreement with already published data. 


\section{Compliance with ethical standards}

\section{Acknowledgments}

\section{Disclosure of conflict of interest}

All authors have no conflict of interest.

\section{Author's contribution}

S.B. Conceptualization, mythology, software, resources, investigation, writing-original draft preparation and validation. C.A. Formal analysis and validation.

L. E. Formal analysis, writing-review and editing and validation.

\section{REFERENCES}

[1] FW Grover. Inductance Calculations, New York: Dover. 1964.

[2] Chester Snow. Formulas for Computing Capacitance and Inductance, National Bureau of Standards Circular 544, Washington DC. December 1954.

[3] PL Kalantarov, et al. Inductance Calculations, National Power Press, Moscow. 1955.

[4] Coulomb JL, G Meunier. Finite element implementation of virtual work principle for magnetic and electric force and torque computation, IEEE Trans. Mag. 1984; 20(5): 1894 -1896.

[5] Benhamou A, AC Williamson, ABJ Reece. Force and torque computation from 2-D and 3-D finite element field solutions, IEE Proc. - Electr. Power Apll. 1999; 146(1).

[6] Tarnhuvud T, K Reichert. Accuracy problems of force and torque calculation in FE systems, IEEE Trans. Magn. January 1988; 24(1): 443-446.

[7] A. Bossavit. Complementarity bilateral bounds on forces in magnet systems, COMPEL-The international journal for computation. 2017; 26(4): 932-940.

[8] Coulomb JL. A methodology for the determination of global quantities from a finite element analysis and its applications to the evaluation of magnetic forces, torques and stiffness, IEEE Trans. Magn. November 1983; 19(6): 2514-2519.

[9] KB Kim, E Levi, Z Zabar, L Birenbaum. Restoring force between two noncoaxial circular coils, IEEE. Trans. Magn. Mar. 1997; 32(2): 478-484.

[10] EP Furlani. A formula for the levitation force between magnetic disks, IEEE Transactions on Magnetics. Nov. 1993; 29(6): 4165-4169.

[11] EP Furlani. Formulas for the force and torque of axial couplings, IEEE Transactions on Magnetics. Sep. 1993; 29(5): 2295-2301.

[12] Y Ren, F Wang, Z Chen, W Chen. Mechanical stability of superconducting magnet with epoxy impregnated, J. Supercond. Novel Magn. 2010; 23: 1589-1593.

[13] Y Ren et al. Magnetic force calculation between misaligned coils for a superconducting magnet, IEEE Trans. Appl. Supercond. December 2010; 20(6): 2350-2353.

[14] SI Babic, C Akyel. Calculating mutual inductance between circular coils with inclined axes in air, IEEE Trans. On Mag. July 2008; 44(7): 1743-1750.

[15] Conway JT. Analytical and Semi-Analytical Solutions for the Force between Circular Loops in Parallel Planes, IEEE Trans. On Mag. 2013; 48(8).

[16] JT Conway. Exact solutions for the mutual inductance of circular coils and elliptic coils, IEEE Trans. On Magn. 2012; 48(1).

[17] Conway JT. Force between Thin Coils with Parallel Axes Using Bessel Functions, IEEE Trans. On Magn. Sept. 2013; 49(9). 
[18] S Babic, C Akyel, Y Ren, W Chen. Magnetic Force Calculation between Circular Coils of Rectangular Cross Section with Parallel Axes for Superconducting Magnet, Progress in Electromagnetics Research B. 2012; 37: 275-288.

[19] SI Babic, C Akyel. Magnetic Force between Inclined Circular Loops (Lorentz Approach), Progress in Electromagnetics Research B. 2012; 38: 333-349.

[20] S Babic, C Akyel. Magnetic force calculation between thin coaxial circular coils in air, IEEE. Trans. Magn. Apr. 2008; 44(4): 445-452.

[21] M Abramowitz, IA Stegun. Handbook of Mathematical Functions, National Bureau of Standards Applied Mathematics, Washington DC. December 1972; 55: 595.

[22] IS Gradshteyn, IM Rhyzik. Tables of Integrals, Series and Products, Dover, New York. 1972. 